\title{
Involuntary pseudos. The untransposed stereos in the digital archives
}

\author{
Victor Flores, Universidade Lusófona, Lisboa, Portugal
}

\begin{abstract}
Digitizing stereo photographs can bring back to museums and archives some of the challenges that early photographers had to deal with. When digitizing the original negative plates in wet collodion or in gelatin silver, the protocol followed for stereos is apparently the same as for mono photography. As a result, archives are presenting to their public and researchers files with untransposed left and right images of each stereo pair. In other words, wrong or 'pseudo views' are being uploaded daily in institutional websites and databases, adding an extra layer of difficulty for the presentation and viewing of some historical gems of western visual culture. This problem was first noticed during a research project dedicated to Portuguese stereo photography which has catalogued thirty one collections of stereo photography, consisting of mainly negatives.
\end{abstract}

Forgotten or neglected media can contribute to the knowledge of current media. Such is the leit-motiv of the recent field of studies named media archaeology (Huhtamo and Parikka, 2011). In the history of photography there is a technique called 'stereoscopy' which was forgotten and largely neglected, and that could contribute to our understanding of current immersive environments of virtual reality. This was the motivation for a research project dedicated to the study of Portuguese stereo photography, which was undertaken between 2013 and 2016. Since there had been no previous studies in this field in Portugal, this project aimed at identifying and cataloguing thirty one public collections of stereo photography in nine different archives and museums. A total of 11.453 images were studied and uploaded in a database, offering us a representative view over the Portuguese Stereo Visual Culture (the title of our project) from the 1860 's to the 1920 's ${ }^{1}$.
One of the main conclusions reached by the project was that about $80 \%$ of the collections kept by the Portuguese public museums and archives were estates of numerous amateur photographers (only about $20 \%$ were commercial collections) with a regrettable but quite expectable feature: most of the photographs were in their original negative format. Since the stereoviews needed to be observed properly, i.e., in stereo, that fact would not have been a setback if only these negatives had been digitized and prepared correctly.

Our goal was to view and to analyse these images in side by side 3D TV screens or in 3D projections and to later include them in an exhibition. (Figure 1) ${ }^{2}$. However, we have quicky concluded that a crucial step had been skipped in the digitization process: the transposition of the right and left images. This is an important and forgotten detail in stereo photography whenever a single plate receives both images in the camera: since right and left images are upside down, every time the plate is inverted, a transposition of both images is required so that the right image corresponds to the right eye, and the same for the left one. This was a common operation when preparing stereo prints in the 19th century. The same transposition had to be applied to stereo direct positives, although implicating a more drastic operation: daguerreotypes, tintypes and, later, autochromes had their plates literally cut and then transposed to guarantee a proper stereopsis.

Without such transposition the depth effects were reversed and pseudoscopy, or a 'view with an error', occured giving a bad and mostly painful experience ${ }^{3}$. The inconvenience of cutting the plate in two halves has lead Jules Richard (1848-1930), the French inventor of the Verascope stereo camera, to invent an inverter stereoscope, a complex optical device originally called 'stereo rédresseur' (Figure 2) that enabled the correct observation of untransposed plates, namely autochrome plates with Verascope format $(45 \times 107 \mathrm{~mm})^{4}$. Such knowledge was lost in the archives that included stereo photography in their

\footnotetext{
${ }^{1}$ A preliminary short edition of the project's book has been published in 2016. See online Flores, Victor (ed.), The Third Image. The Stereo Photography in Portugal, Documenta. A short summary of the project can also be found at: https:// stereovisualculture.ulusofona.pt

2 The exhibition was entitled The Third Image. The Stereoscopic Photography in Portugal and the Desire for 3D. It was curated by Victor Flores, Ana David and Sofia Castro and took place in three different venues during 2015: at the Museum of Moving Image, in Leiria, at the Archivo Municipal de Lisboa - Fotográfico, and at the National Archive of Torre do Tombo, in Lisbon.

${ }^{3}$ Pseudoscopy was an optical imperfection noticed in the first binocular microscopes. It was later revived by Charles Wheatstone in 1852 by describing the pseudoscope in his Contributions to the physiology of vision - Part the second as an instrument that allowed the conversion of relief 'exhibited by binocular pictures in the stereoscope, when they are transposed, reflected or inverted' ( $\$ 23)$. The term 'pseudoscopy' designates the illusions or false perceptions that invert depth relations and transform concave objects like a tea-cup into a 'solid convex body'. As Wheatstone stated: 'With the pseudoscope we have a glance, as it were, into another visible world, in which external objects and our internal perceptions have no longer their habitual relation with each other.' (\$23) The pseudoscope helped Wheatstone to study and to manipulate binocular disparities in the stereoscope.
}

${ }^{4}$ This ingenious device combined a tetrahedral prism with other prisms and was described by Jules Richard and E. Colardeau in the paper 'Sur la stéreoscopie pseudoscopique' in 1916. 
holdings, bringing an extra difficulty in studying and in presenting stereoviews to the public.

Today it is crucial to reflect on the reasons for this ocurrence. This specialized knowledge got somehow lost in this process not only because stereoscopic photography was discontinued throughout the 20th century but also because archives and museums were eager to maximize digital coverage of their collections. Since the 90's digital tools became the touchstone of the equipments in the workflow of the archives. With inexpensive softwares it became possible to have the positive version of a negative without any prints. The positivation of the negatives was part of the scanning process in any archive. As a result, negatives became more useful (and valuable!) to archives. The positivized files could be presented in their websites and made avaiable to curators and researchers. On the other hand, stereoscopic negatives were included in the scanning workflows along with the monoscopic photographs. Since no distinction was made between these two different types of negatives, no transpositions between left and right image were planned. Involuntarely, this process has converted stereoscopic negatives in pseudoscopic digital files which have been published online, printed in catalogues and exhibited in museum walls.

The urge to ensure a quick digitization of their collections combined with a lack of knowledge about this quite 'archaeological process' has provoked an inappropriate approach for scanning and editing stereoscopic negatives in most Portuguese archives and museums. Although these digitizations have promoted remote access to the collections and protected the fragile items from handling, they didn't necessarily promote stereoscopic photography. To a certain extent, this digitization approach contributed to the repression of stereo photography as a different and very specific photographic technique. It is crucial to acknowledge that digitization should contribute to the scholarship on topics such as this one, instead of following the standards of monoscopic photography. As Andrew Prescott and Lorna Hughes (2018) have recently stated: 'If digital libraries merely reiterate and reinforce long-standing cultural narratives and stereotypes, rather than enabling the exploration of forgotten and neglected collections, then they can become agents of cultural exclusion.'

Stereoscopic negatives would definetly benefit from the 'slow digitization' recommended by these two authors. Imbued with a critical framework that promotes the reflection on how the digitization is undertaken and how it can support scholarship, this 'slow digitization' aims to foster deeper and richer educational experiences. Although inspired by a need for a deeper examination of manuscripts, it would surely help to rethink the digitization of stereo negatives.

We do know for a fact that properly transposed stereos cannot provide the intended stereoscopic vision in the computer screen unless anaglyphic or gif techniques are used. However, not transposing the exposures adds a layer of difficulty and misunderstanding to the process. For the comparative study and analysis of these collections spread in Portugal (from Oporto to Azores), the use of digital files proved to be rather important. An intermediate post-production phase for photo edition was added to our workflow. In order to deliver perfect and eye comfortable stereo effects it took us three steps: first, left and right images were transposed in each file; secondly, the images were cropped to make sure the visible areas were common in both images. By trimming each image and reducing to a squared center, the tilting effects in the edges of the stereoviews were supressed. This was a common gesture in stereo photography back in the 19th century. In one of the estates included in our research we have found nineteen negative glass plates $(12$ X 20,5 cm) by Emílio Biel, a German photographer who owned an important photographic studio in Oporto, which had the emulsions scratched with thin lines composing squares that marked the proper framing for the print crop. These plates (Figure 3) were immediately assumed as a reference for a semiautomatic cropping in large photographic estates such as the one of Aurélio da Paz dos Reis, at the Portuguese Center of Photography, which included 7296 untransposed negatives, or the Afonso Chaves estate in Museu Carlos Machado in Azores, with around 5879 untransposed negatives (Figures 4 and 5).

Finally, the third step, also undertaken in Photoshop, ensured the horizontal and vertical alignment of the images by using a simple grid that simplified the matching of both images (Figure 6). Unfortunately no automation could be employed to accomplish a proper positioning of the square since it can be aligned in different heights depending on the photo composition. Emílio Biel's negatives present this same situation: the lines were scratched either next to the top or to the bottom depending on the subject and on the composition. This step can be described as the stereo photographer's 'final cut' (a literal one!) since it is an aesthetic decision that will emphasize his compositions and enhance stereo effects.

This research on Portuguese stereoscopic photography was recently followed by another project on the stereo photography by Carlos Relvas (1838-1894), one of the most renowned early Portuguese photographers whose stereoviews had not yet been studied either. All the 637 stereos of his estate at the Studio House Carlos Relvas, in Golegã, are negatives and their digital files present the same issue. As for the previous project, we have prepared these images for an exhibition at the National Museum of Chiado 5 , in Lisbon, where a stereo projection room showcased these negatives properly, contributing to the dissemination of these images and reconstituting their effects lost 150 years ago. Portugal is certainly not the only example of these situations, and considering stereo archives are an important source of historical and medialogical information, a norm or recommendation should be planned to prevent these errors and raise awarenesss on this topic. A better adjustment is needed between the analog plate and the digital file to be used by the institution and experienced by the public. Pseudos shouldn't be the norm in the digital files that have rescued the stereoscopic negative plates.

5 The exhibition was entitled 'Carlos Relvas (1838-1894)— Rediscovered Views of Portugal. Photography in the European Salons' . It took place from September 2018 to February 2019 in Museu do Chiado (Lisbon) and was curated by Victor Flores, Ana David, Denis Pellerin and Emília Tavares. 


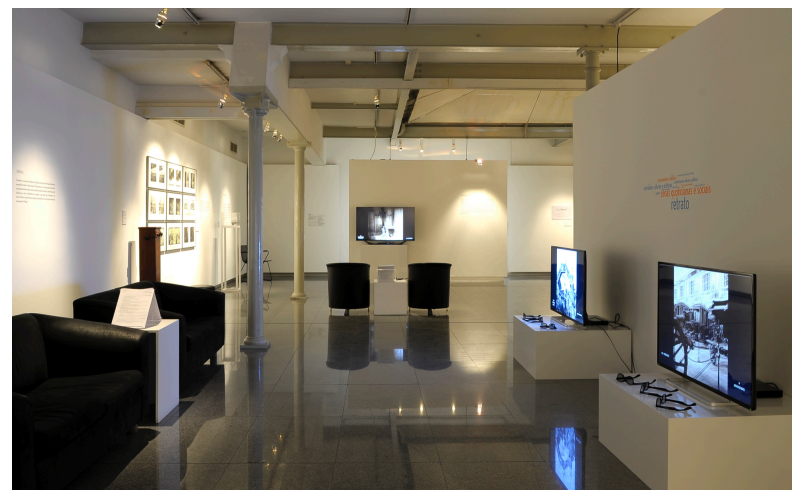

Figure 1. A view from the exhibition The Third Image presenting stereoviews in TV sets along with 19th century stereo viewers (Arquivo Municipal de Lisboa- Fotográfico)

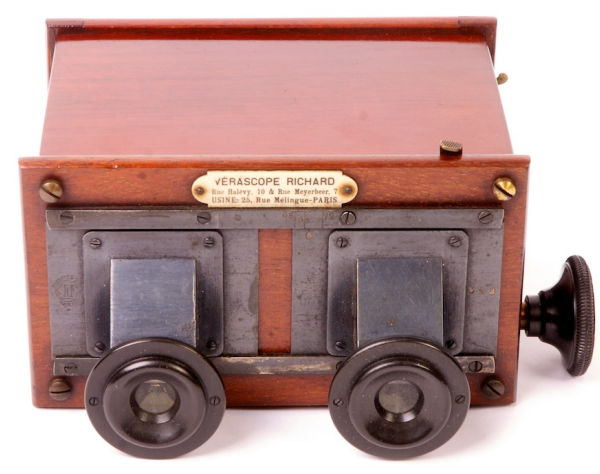

Figure 2. Inverter stereoscope or 'stereo redresseur' by Jules Richard, c. 1915

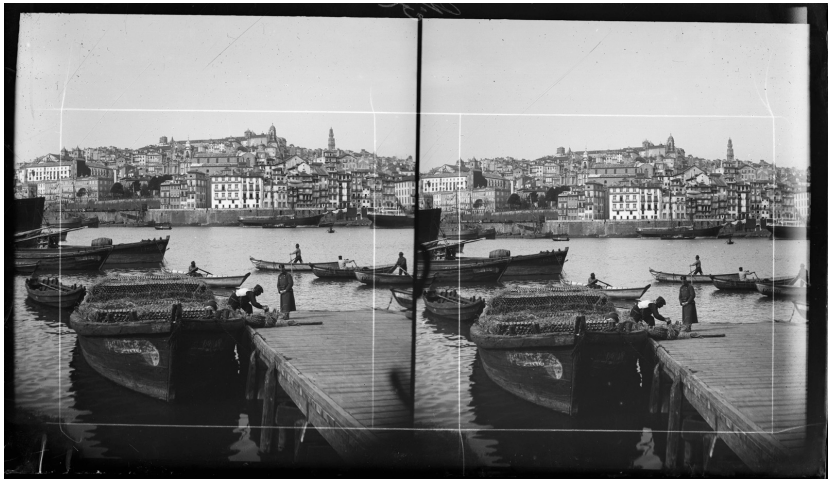

Figure 3. Emílio Biel, [boats anchored in Cais da Ribeira, Oporto], untransposed stereoscopic negative plate, silver gelatin, ca. 1900, Science Museum, Oporto University.

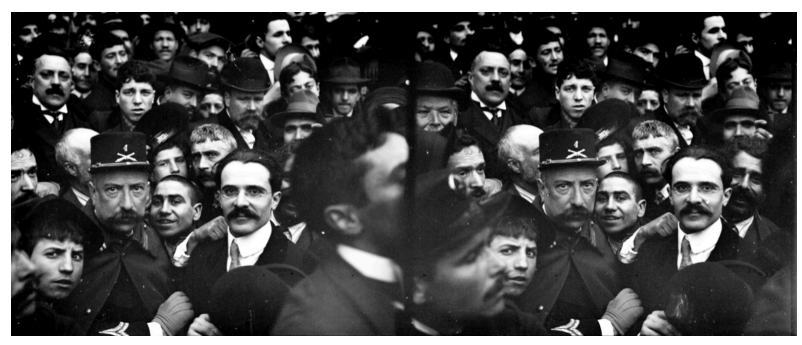

Figure 4. Aurélio Paz dos Reis, [Waiting for Afonso Costa], untransposed stereoscopic negative plate, silver gelatin, ca. 1910 @) Centro Português de Fotografia, DGLAB-MC.

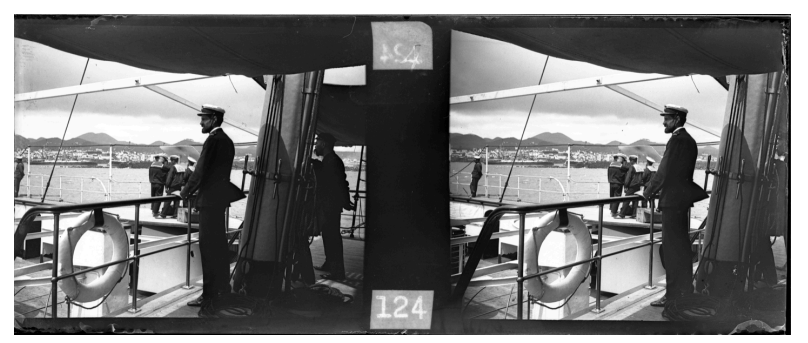

Figure 5. Francisco Afonso Chaves, Untitled, untransposed stereoscopic negative plate, silver gelatin, c. 1900, Museu Carlos Machado.

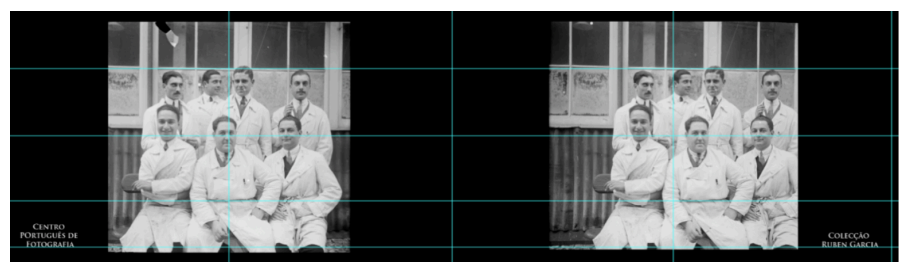

Figure 6. Vertical and horizontal alignment of stereoscopic photos in Photoshop 


\section{References}

[1] Erkki Huhtamo; Jussi Parikka, Media Archaeology. Approaches, Applications and Implications, Berkeley and Los Angeles, California, University of California Press (2011).

[2] Andrew Prescott; Lorna Hughes, 'Why do we digitize? The case for slow digitization', Archive Journal, September (2018).

[3] Charles Wheatstone, 'The Bakerian Lecture. Contributions to the Physiology of Vision - Part the Second. On some remarkable, and hitherto unobserved, Phenomena of Binocular Vision (continued)' in Philosophical Transactions, The Royal Society Publishing (1852).

\section{Author Biography}

Victor Flores is an Associate Professor at Universidade Lusófona in Lisbon, and a visual media researcher at The Centre for Research in Applied Communication, Culture and New Technologies (CICANT). He is the founding organizer of the International Conference on Stereo \& Immersive Media: Photography and Sound Research, and is the

principal editor of its corresponding journal. He has been coordinating research projects since 2012, mostly related to stereo photography, and has curated several exhibitions dedicated to early stereo photographers. 